Ophthalmologe $2017 \cdot 114: 772-774$

DOI 10.1007/s00347-017-0542-7

Online publiziert: 18. August 2017

(c) Springer Medizin Verlag GmbH 2017

CrossMark

\section{T. Kohnen ${ }^{1,2}$}

${ }^{1}$ Klinik für Augenheilkunde, Goethe-Universität, Frankfurt am Main, Deutschland

${ }^{2}$ Cullen Eye Institute, Baylor College of Medicine, Houston, USA

\title{
Deutsche Augenheilkunde international
}

Die deutsche Ophthalmologische Gesellschaft (DOG) wurde im September 1857 von Albrecht von Graefe (1828-1870) und 11 Gleichgesinnten gegründet und gilt damit als die älteste augenärztliche Gesellschaft weltweit. Von Graefe hatte neben seinem wissenschaftlichen Interesse vor allem eine Persönlichkeit, die ihm höchstes nationales, aber auch internationales Ansehen einbrachte. Sowohl bei seinen Gründungen, des Archiv für Ophthalmologie 1854, der ersten ophthalmologischen Fachzeitschrift weltweit, die heute noch existiert, und der (Deutschen) Ophthalmologischen Gesellschaft (DOG) 1857 als auch in seiner eigenen damals weltberühmten Klinik in Berlin spielten ausländische Kollegen und Patienten eine große Rolle, wie Rohrbach in dem ersten Beitrag zum Leitthema dieser Ausgabe von Der Ophthalmologe aufzeigt.

Man setzt den Beginn der modernen Augenheilkunde mit der Erfindung des Augenspiegels gleich. Von da an hat die deutsche Augenheilkunde die internationale Entwicklung unseres Fachs mit beeinflusst. Denke man nur nach der Entwicklung des Augenspiegels (durch von Helmholtz), an die Photokoagulation (Meyer-Schickerath), die Kapsulorhexis (Neuhann) oder erst kürzlich das Retinaimplantat (Zrenner) - Erfindungen und operative Maßnahmen aus Deutschland, die weltweit die Augenheilkunde weitergebracht und verändert haben (Müller et al.).

Eine sehr einschneidende Zeit in der deutschen Augenheilkunde wird von Manfred Jähne beschrieben, der die internationalen Beziehungen der Augenheilkunde in der ehemaligen DDR in seinem Beitrag beleuchtet. Jähne beschreibt anhand eigener Erfahrungen, wie durch massive Einschränkungen die nationalen, innerdeutschen und internationalen Beziehungen der Augenheilkunde in der ehemaligen DDR vehement behindert wurden.

\section{\) Seit Erfindung des Augen- spiegels hat die deutsche Au- genheilkunde die internationale Entwicklung beeinflusst}

Heute ist die weltweite Vermeidung der Blindheit eins der großen Themen in der Augenheilkunde. So wurde im Jahr 1999 die globale Initiative „Vision 2020 - The Right to Sight“ als ein Kooperationsprojekt der World Health Organization (WHO), der International Agency for the Prevention of Blindness (IAPB) und mehrerer Nicht-Regierungsorganisationen (NGOs) gegründet. Diese vorbildliche Idee hat weltweit zum intensiveren Engagement für die Blindheitsverhütung geführt. In der DOG wurde 2004 die seit 1994 bestehende Arbeitsgruppe „Internationale Ophthalmologie“ in die gleichnamige Sektion umgewandelt. Nentwich et al. schreiben in ihrem Beitrag über die schon zuvor etablierten Partnerschaften deutscher und afrikanischer Augenkliniken, durch die diese Kliniken zu wesentlichen Zentren augenärztlicher Weiterbildung und klinischer Versorgung in Ostafrika wurden. Mittlerweile existieren in Deutschland nach diesem Vorbild eine Reihe von ähnlichen Klinikkooperationen und eine Vielzahl weiterer Projekte.

In der letzten Arbeit dieses Leitthemas steht die internationale Vernet- zung der augenheilkundlichen Grundlagenforschung in Deutschland im Fokus. Experimentelle Basisforschung bildet die Grundlage für das Verständnis pathophysiologischer Krankheitsprozesse und die Entwicklung neuer Diagnose- und Therapieansätze augenheilkundlicher Erkrankungen. Schlötzer-Schrehardt und Cursiefen zeigen in ihrem Beitrag, dass die ophthalmologische Grundlagenforschung in Deutschland hauptsächlich in den Universitätsaugenkliniken betrieben wird. Als Drittmittelgeber für augenheilkundliche Forschungsprojekte spielen vor allem die Deutsche Forschungsgemeinschaft (DFG), das Bundesministerium für Bildung und Forschung (BMBF) und zunehmend auch die Europäische Union (EU) eine große Rolle. Die ophthalmologische Grundlagenforschung ist daher sowohl in zahlreiche europäische Forschungsnetzwerke eingebunden als auch darüber hinaus in vielfältiger Weise international vernetzt. Trotz der hiesigen, im Vergleich $\mathrm{zu}$ den USA relativ bescheidenen finanziellen Möglichkeiten ergibt sich ein vergleichsweise hoher wissenschaftlicher Output der deutschen Augenforscher. In Anbetracht des rasanten Aufschwungs asiatischer Länder besteht jedoch der dringend notwendige Handlungsbedarf, die Wettbewerbsfähigkeit Deutschlands und seiner Leistungsträger zu stärken und für die Zukunft im internationalen Vergleich zu sichern.

Ganz besonders im Zeitalter der Globalisierung ist eine enge Vernetzung in Wissenschaft, Lehre und Krankenversorgung, eine Betrachtung unseres Faches aus unterschiedlichen Blickwinkeln sowie ein fruchtbarer Erfahrungsaustausch und Wissenstransfer auf internationaler 
Hier steht eine Anzeige.

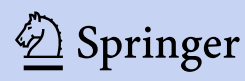


Ebene unverzichtbar geworden. Schwerpunktthemen für den DOG-Kongress 2017 mit dem Titel „Deutsche Augenheilkunde international“ sind daher unter anderem in Gebieten der Augenheilkunde zu suchen, in denen deutsche Forschung einen entscheidenden Anteil an der internationalen Entwicklung gehabt hat und hat.

Ich möchte mich bei allen Mitgliedern der DOG für ihr Vertrauen und die Möglichkeit, im Jahrgang 2016/2017 ihr Präsident sein zu dürfen, bedanken. Ich wünsche Ihnen bei der Lektüre dieser fünf Arbeiten viel Freude, einen guten Wissensgewinn und hoffe, Ihnen mit dem Thema „Deutsche Augenheilkunde international" und der Zusammenstellung der Arbeiten in dieser Ausgabe des Ophthalmogen einen Einblick in die Vergangenheit und in interessante Entwicklungen für Gegenwart und Zukunft unseres Fachgebiets gegeben zu haben.

\section{Herzlichst}

Ihr

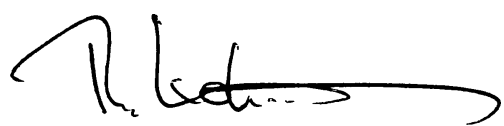

Univ.-Prof. Dr. Thomas Kohnen Präsident der DOG

\section{Korrespondenzadresse}

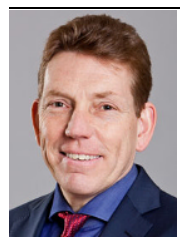

Univ.-Prof. Dr. T. Kohnen

Klinik für Augenheilkunde, Goethe-Universität

Theodor-Stern-Kai 7, 60590 Frankfurt am Main, Deutschland Kohnen@em.uni-frankfurt.de

Interessenkonflikt. T. Kohnen gibt an, dass kein Interessenkonflikt besteht.

\section{Immer aktuell - dank „Online-First“-Publikation}

Warum warten, bis die Artikel im Heft abgedruckt sind, wenn Sie sie auch schon vorher lesen können?

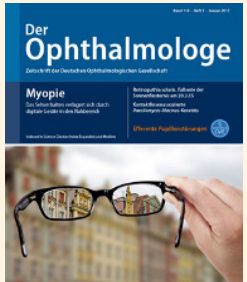

Lesen Sie unter www.springermedizin.de/der-ophthalmologe "Online First Artikel” die aktuellsten Artikel, die gerade online veröffentlicht wurden.

Folgende Übersichten, Originalien, Kasuistiken und „Bild und Fall“Beiträge wurden im vergangenen Monat online publiziert:

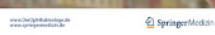

- Intravitreales Methotrexat zur Behandlung eines uveitisch bedingten zystoiden Makulaödems.

J. Maaß, E. Matthé. doi:10.1007/s00347-017-0548-1

- Maximal weite lichtstarre Pupille und primäre Transplantatinsuffizienz nach perforierender Keratoplastik.

G. SchießI, T. Hager, B. Seitz. doi:10.1007/s00347-017-0553-4

- Akuter monokularer Visusverlust.Differenzialdiagnostische Überlegung abseits der internistisch-ätiologischen Abklärung.

A. Rickmann, M. A. Macek, P. Szurman et al. doi:10.1007/s00347-017-0555-2

- Trends in der Ablatiochirurgie. Was hat sich im Vergleich zu 2001 geändert? C. V. Fischer, M. Kulanga, H. Hoerauf. doi:10.1007/s00347-017-0559-y

- CyberKnife $^{\circledR}$ : Eine neue Therapieoption bei uvealem Melanom. J. M. Mor, R. Semrau, W. Baus et al. doi:10.1007/s00347-017-0560-5

Übrigens: Wussten Sie schon, dass die Artikel mit Angabe der DOI (digital object identifier) und dem Zusatz "epub ahead of print" bereits zitiert werden können? 